\title{
Are good ideas enough? The impact of socio-economic and regulatory factors on GMO commercialisation
}

\author{
Núria Vàzquez-Salat ${ }^{1}$ \\ ' Global Biopolitics Research Group, Department of Political Economy, King's College London, Strand, London WC2R 1HH, United Kingdom
}

\begin{abstract}
In recent years scientific literature has seen an increase in publications describing new transgenic applications. Although technically-sound, these promising developments might not necessarily translate into products available to the consumer. This article highlights the impact of external factors on the commercial viability of Genetically Modified (GM) animals in the pharmaceutical and food sectors. Through the division of the production chain into three Policy Domains -Science, Market and Public- I present an overview of the broad range of regulatory and socio-economic components that impacts on the path towards commercialisation of GM animals. To further illustrate the unique combination of forces that influence each application, I provide an in-depth analysis of two real cases: GM rabbits producing human polyclonal antibodies (pharmaceutical case study) and GM cows producing recombinant human lactoferrin (food case study). The inability to generalise over the commercial success of a given transgenic application should encourage researchers to perform these type of exercises early in the R\&D process. Furthermore, through the analysis of these case studies we can observe a change in the biopolitics of Genetically Modified Organisms (GMOs). Contrary to the GM plant biopolitical landscape, developing states such as China and Argentina are placing themselves as global leaders in GM animals. The pro-GM attitude of these states is likely to cause a shift in the political evolution of global GMO governance.
\end{abstract}

Key words: Genetically modified organisms, GM animals, policy domains, governance, socio-economic factors

\section{INTRODUCTION}

When developing a new scientific application, researchers acknowledge the importance of a solid research design and the consideration of possible bottlenecks for their project. Conversely, this careful planning is not always applied when the application aims to reach the market. Most researchers may only consider the end user and the regulatory set up in which their application would navigate. Unfortunately, these factors alone might not guarantee its commercial success. There are a series of regulatory and socio-economic variables that will determine the market permeability of a given biotechnology application.

Genetically Modified (GM) plants are an excellent example of a technology that could not reach its full potential as a result of the influence of these forces. Despite the similarity of the topics debated worldwide, the presence of a variety of citizens' values and different interest groups triggered a broad range of policies towards GMOs (Vàzquez-Salat et al., 2012). The resulting global GMO governance had major implications for the commercial prospects of succeeding GM plant applications, as well as other applications using the same technology. GM animals, for example, are only slowly reaching the commercialisation stage despite starting at the R\&D stage at similar time as GM plants (Vàzquez-Salat and Houdebine, 2013). Their GMO status requires them to fit in a crumblesome and expensive regulatory set up, making them an excellent example to study the influence of external forces in the commercialisation of scientific applications. Indeed, their ability to reach the end-user will not only be influenced by their intrinsic characteristics but also, by the result of a highly controversial GMO debate that took place twenty years ago.
Using GM animals as a vehicle I describe the barriers specific or not to their GMO status - that might arise before reaching the end user in the two most important commercial routes: the pharmaceutical and food sectors. The array of interest groups and issues that each application can trigger is likely to be unique, and might even show regional variation. To highlight this complexity, I provide a detailed analysis of two case studies from the existing pipeline of GM animal applications. These applications -one pharma and one foodwhere chosen by the PEGASUS Consortium based on their probability to reach the market and expected impact on the end user (www.wageningenur.nl/ en/project/Pegasus.htm).

\section{EXTERNAL FACTORS THAT WILL SHAPE THE COMMER- CIALISATION OF GM ANIMALS}

Any particular scientific application is subject to a number of internal and external influences that determines its penetrance to the market. Technical bottlenecks are a good example of internal factors that can delay the commercialisation of biotech applications. GM animals for example, were developed at the same time as GM plants but took longer to reach the market due to early inefficient laboratory techniques and biological complexity of animals (Vàzquez-Salat and Houdebine, 2013). Nonetheless, once a GM animal (or its products) leaves the laboratory premises, it still needs to interact with an array of external factors. It is important to identify from the early stages the range of components that are likely to impact on the commercialisation of a given application. These can also vary across regions and may include the regulatory set up, market demand or cultural values, amongst others. These forces can be difficult to disentangle because they might vary at 
regional level, act in synergy or compensate for each other. The different characteristics and interactions among these variables will produce a unique medium, which will ultimately condition the commercialisation of GM animals.

Since an application leaves the laboratory until it reaches the end user, it will navigate through a variety of environments across the production chain. The different type of stakeholders, regulatory landscapes and nature of the sector will create a set of intrinsic characteristics that will define each domain. Known as Policy Domains, these can be subdivided in Science, Market and Public (Vàzquez-Salat and Houdebine, 2013). The Science Domain covers the R\&D stage and it is characterised by a high level of homogenisation. This globalisation of practice is promoted through international collaborations and a strong culture of standardisation (Royal Society, 2011). Indeed, only in the initial steps of R\&D -dominated by public research- there is high level of convergence between the pharma and food sectors (Bagchi-Sen and Scully, 2007).

The Market Domain is specific to the industry, with a varying degree of globalisation and number/type of stakeholders involved. The breeding sector has a high number of stakeholders throughout the production chain although it varies according to the species. It can range from the family-run farm model of the poultry industry to strong the vertical integration observed in the aquaculture sector (Neeteson-van Niewenhoven, 1999). Conversely, the pharmaceutical sector -dominated by Multi-National Corporations (MNC)- is strongly vertically integrated. The final stakeholders of the food Market Domain, producers and retailers, are particularly sensitive to consumer's preferences. Strongly hit by the 90 s GMO crisis, they display the highest level of resistance to GM animal products compared to other stakeholders. In contrast, their counterparts in the pharmaceutical sector were never exposed to the anti-GM campaigns in the 90s. As a result, the GMO status of a given application is never taken into consideration when considering its commercial prospects (Vàzquez-Salat and Salter, 2011).

The third Domain -the Public Domain - includes citizens as well as numerous interest groups such as environmental nongovernmental organisations (NGOs), animal welfare groups or media. This Domain is not only highly heterogeneous, but also varies across sectors and world regions. For example, consumers' priorities between developing and developed states change dramatically (Vàzquez-Salat et al., 2010). This Domain played a major role in shaping the GM plant debate in the 90s, and as expected, it was also identified as the major limiting step for GM animals (Vàzquez-Salat and Salter, 2011). The industry's different attitude towards GMOs in the Market Domain can be attributed to the type and interests of the end user in the pharma and breeding sectors. Whilst the food sector targets the consumer -a highly heterogeneous group with a broad range of prioritiesthe pharmaceutical sector caters for patients. The patient community, with strong vested interests in these applications and a higher willingness to take risks, is committed to innovation. Thus, whereas GM applications in the pharma sector are not expected to encounter any resistance, their adoption in the food sector is envisaged to have a patchy pattern as a result of regional variation in both, ethical and cultural values.

\section{BRINGING THEORY INTO PRACTICE: THE CASE STUDIES}

Using two real examples -a pharmaceutical and a food case studies- I highlight the interaction of different internal and external forces described in the previous section throughout the production chain. Some of the characteristics described in this section are general to GM animals or to their biotechnology nature, whereas others are unique to a given application. All of these factors ought be taken into account at the R\&D stage due to their expected impact later in the commercialisation path. The goal of this type of exercise is to facilitate the understanding of the specific barriers that scientific applications are likely to encounter before reaching the market (if they reach it). Furthermore, in controversial fields such as GM technology, performing these exercises would enable the early identification of future bottlenecks (e.g. animal welfare, consumer rejection, market competition, etc.).

\subsection{The pharma case: GM rabbits producing human polyclonal antibodies}

As a result of some recent technical improvements which have eliminated unwanted side effects, monoclonal antibodies are currently an important class of pharmaceutical products (Buelow and van Schooten, 2007). The existing market is highly profitable, with revenues around \$300-350 million (Vàzquez-Salat and Salter, 2011). Under such an encouraging economic scenario and a clear market need, it is not surprising that several research teams have tried to develop bioreactors producing human polyclonal antibodies. Although technically more challenging, polyclonal antibodies -able to recognise multiple epitopes- have higher therapeutic activity than their monoclonal counterparts (Mora et al., 2011).

There are currently three companies that use GM animals as production platforms for these antibodies are moving towards the commercialisation stage. Each one of these companies focuses on a different species: cows by Hematech (USA), pigs by University of Pittsburgh (USA) and rabbits by Therapeutic Human Polyclonal or THP (Germany). There are advantages and disadvantages for each of these species and thus, the final choice will depend on market preferences such as the quantity of the final product needed (VàzquezSalat and Salter, 2011). In this section I discuss the Therapeutic Human Polyclonal (THP) case because it will likely find a smother regulatory path. Compared to the other GM animals, rabbits do not suffer diseases that can infect humans (e.g. prion diseases) and regulatory bodies already have extensive information on the species' basic biology (Vàzquez-Salat and Salter, 2011).

Developed by a German subsidiary of THP Inc., these GM rabbits contained human IgG levels up to $2 \mathrm{mg} / \mathrm{ml}$ of blood (Buelow and van Schooten, 2007). Unfortunately, the knock out of these rabbit IgG genes was partial. As the animals grew up their B cells showed a preference for the rabbit heavy chain compared to the human one. By the time the rabbits were mature enough to extract antibodies from them, their B cell populations produced only rabbit antibodies (Vàzquez-Salat and Salter, 2011). At that stage the application required further troubleshooting, or in other words, more funding. However, the Venture Capital company behind THP Inc. was not willing to invest further in the model and sold the company to Roche in 2007. A global leader in biologic therapies, Roche was only interested in using the TPH rabbit model for the production of monoclonal antibodies. Thus, following the change of ownership, the human polyclonal project was terminated (Vàzquez-Salat and Salter, 2011). 
Even if Roche had supported the development of these GM rabbits for the production of human polyclonal antibodies, this application would not remain free of constraints. Two major bottlenecks -economic and regulatory- were identified at the Market Domain. Although this situation might be overcome in the future as transgenic techniques improve, currently most of the costs of producing polyclonal antibodies lie in downstream manufacturing (i.e. purification, sterility, quality control). It has been estimated that $90 \%$ of the costs of producing complex proteins are due to the purification stages and only $10 \%$ on production ones (Mora et al., 2011). Even if using GM animals for the production of antibodies is cheaper in the long term, they are not an attractive economic option compared to existing cell culture platforms (i.e. $\mathrm{CHO}$ cell cultures). In terms of regulatory bottlenecks, bodies such as the FDA and EMA are known for posing significant safety constraints on the life science innovation system. For example, the FDA requires that any product should be free of animal material to avoid the risk of zoonoses. When your production platform is an animal -as it is the case of THP rabbits- it is impossible to compile with this requirement.

Pharmaceutical companies are known to push for heavy regulations in order to retain the dominance of their existing markets (Vàzquez-Salat and Salter, 2011). There is a significant amount of economic investment behind the development of each therapeutic product. To guarantee the success of their products, companies invest much of their R\&D in predictable and manageable path-dependent innovations (Tait, 2007). Added to the competitiveness of the healthcare market, big pharmaceutical companies will be reluctant to move to new avenues. For this case study for example, Roche had no intention to move to the polyclonal field after having achieved global leadership in the monoclonal market (VàzquezSalat and Salter, 2011). Moreover, this specific application could be described as a potential disruptive innovation (Tait, 2007): the immune response triggered by polyclonal antibodies is stronger than the one produced by monoclonal antibodies. Human polyclonal antibodies would be expected to outcompete the existing monoclonal market. Their introduction could trigger a shift within the existing market with a possibility to create new industry sectors or cause market restructuration. Unfortunately, the industry's view contrasts with the patient's needs in the Public Domain. The benefits of this type of application for the patient community are promising because antibody therapy is used to treat a wide arrange of diseases including cancer or inflammatory disorders. Once THP antibodies reached the market, no resistance would be envisaged at this Domain. Even in antiGM regions such as the EU, medical-related applications receive full support from most interest groups and citizens (Eurobarometer, 2005).

Thus, human polyclonal antibodies produced by GM animals remain at the developmental stage as a result of unexpected technical constraints. Significant investment will be needed at the Science Domain before these bioreactors leave the laboratory. However, as seen in other applications developed by small biotech companies, solving these technicalities might not be enough. Even when they reach the commercialisation stage, they would struggle to find a niche market in the highly competitive healthcare landscape. Despite the novelty of these applications and the expected economic revenues that they might raise, the Market Domain has proven hostile. The resistance to their commercialisation contrasts with the need for these antibodies at the Public Domain. Nevertheless, a number of research teams are currently working on similar projects in a number of species, including rabbits (Houdebine, 2011). The clear market need in conjunction with the promising economic benefits, have encouraged the scientific community to overcome the technical hurdles. The 'pull effect' from the Public Domain acting in synergy with the 'push effect' from the Science Domain might bring this promising application through the Market Domain.

\subsection{Food case: GM cows producing recombinant human lactoferrin $(r h L f)$}

There are several reasons for the selection of this interesting application. First, this is one of the few GMO applications in the food sector which benefits the consumer: Lactoferrin is a glycoprotein that has been shown to have numerous protective properties against various pathogens (Lonnerdal and Bryant, 2006; Tanaka et al., 1999; Troost et al., 2003; Venkatesh and Rong, 2008). Developing GM cows that produce human lactoferrin in their milk would protect vulnerable populations, particularly in developing countries. Second, there are already in the market products containing human or bovine lactoferrin. For example, bovine lactoferrin is being used as food supplement and skin/oral care products (Mora et al., 2011). The production of human lactoferrin is cheaper (higher concentration in the milk) although they have similar purification costs (Mora et al., 2011). Several companies use different production platforms but only three would use GM animals (Mora et al., 2011). In other words, GM animals are an alternative method of production that would have to fit in to a well-established market.

The third interesting point of this case study is its regulatory ambivalence. Recombinant human lactoferrin could be considered as either a drug or a food supplement; following in each case a different set of regulatory frameworks (VàzquezSalat and Salter, 2011). This type of GMO applications have been described as falling in a regulatory "grey area" (Kleter et al., 2001). The authors noted that the increasing interest for nutraceuticals might improve the consumer's attitude towards GMOs. Finally, this application facilitates the comparison between two commercial approaches: Asian versus European. At the time of writing this article there are three groups developing this bioreactor: two of them work with GM cows (Pharming and SKL) and one with GM goats (Institute of Gene Biology) (Mora et al. 2011). Thus, by focusing on GM cows we are able to compare the market approach of Pharming (a Dutch private company) and SKL (a Chinese public research institute).

Pharming is a pharmaceutical biotech company that specialises on innovative therapeutics (www.pharming.com/ index.php?act=corp). With a long history on the development of transgenic animals (Vàzquez-Salat and Houdebine, 2013), they currently have four products under development and one in the market (i.e. Rhucin ${ }^{\circledR} /$ Ruconest $^{\mathrm{TM}}$ ). Conversely, State Key Laboratory for Agrobiotechnology (SKL) at the China Agricultural University (CAU) is one of the laboratories funded by the Chinese government to focus on green biotechnology (www.cuhk.edu.hk/ipmbab/SKL/). GM technology is part of the Chinese government's vision to provide food security throughout the country. The government 
has made significant investments in this field, including for GM animals (Chen and Zhang, 2011). The development of technological applications in China differs from the Western approach. Different laboratories specialise on a specific GM application and collaborate with an 'assigned' biotech company to ensure that the selected application reaches the market (Vàzquez-Salat and Salter, 2011). GM cows producing human recombinant lactoferrin is one of the ten cases of GM animals selected by the government (Vàzquez-Salat and Salter, 2011).

There are some similarities between the European and Asian approaches in several areas. Both teams developed the founder through microinjection and currently manage a herd of GM cows producing recombinant human lactoferrin (rhLf) (Yang et al., 2008; Mora et al., 2011). Their portfolios are moving towards the commercialisation stage and aim to market the product globally (Vàzquez-Salat and Salter, 2011). In both cases, Asian consumers were identified as end users due to their long tradition and cultural understanding of the effects of different foods in health promotion (Vàzquez-Salat and Salter, 2011). The differences appear in the selection of the initial market: SKL would focus in the internal Chinese market whereas Pharming would enter in regions with an established market for lactoferrin, such as Japan (Vàzquez-Salat and Salter, 2011).

As previously stated, rhLf could be marketed as either food supplements or medicines (Kostov et al., 2011). The pharmaceutical regulatory path requires very strict methods of purification but it is compensated with a uniform global regulatory system. For example, one of the companies' strategies to sell these type of products in the USA is through collaborations with the pharma industry (Stein et al., 2008). Nevertheless, both teams chose to sell rhLf as food supplements -as tablets or added to other products- due to the lower level of purification required. This commercialisation pathway though, would expose rhLf to regional cultural and ethical values resulting in a patchy worldwide adoption.

Functional food is a growing market and it has become one of the food industry's priorities (Stein et al., 2008). There are numerous products entering the market every year although only a few consolidate their position. Their success seems to be correlated with cultural and socio-economic factors. For example, the European consumer will look at the qualities of the food whereas the American is more interested in the novelty (Stein et al., 2008). This application is expected to receive higher acceptance in regions where consumers have already shown an interest in the product or where child mortality is a matter of concern. In addition, their commercialisation as tablets might overcome the 'yuck factor' linked to GM animals, even in countries were animal welfare is high on the agenda (e.g. Scandinavian countries). Nevertheless, our results showed that despite its clear benefits, the European consumer was not expected to accept this GM application (Vàzquez-Salat and Salter, 2011). Whereas EU acceptance was one of the cornerstones of the 90s GMO crisis, this time -despite one of the companies being European-it is not a limiting factor. Neither SKL nor Pharming were planning to market their products in the EU, at least in the near future (Vàzquez-Salat and Salter, 2011).

To summarise this case study, several teams are working on this bioreactor at the Science Domain because it is not technically demanding and its product has a well- characterised market. The success of this application thus, is likely to be tested at the Market Domain. As an advantage, GM animals offer a cheaper method of production (although it requires higher investment to develop the founder), low environmental risks and a minimal impact on the animal's welfare. As a disadvantage, the existence of alternative methods of production might render this biological platform redundant. Bioreactors offer only a minimal reduction of costs with most of the expenditure still seen in downstream manufacturing.

In the longer term, both teams aimed to market their products globally although there were some differences in the initial steps. SKL would launch their product in the Chinese internal market whereas Pharming would navigate in a region where lactoferrin is already established. Even though both teams are starting with the same production platform and producing similar quantities of the product, SKL's may enjoy a competitive advantage. They would not only start in a lactoferrin-naïve region but also, the Chinese government might develop a series of regulatory barriers to provide an advantage to their domestic production of rhLf. This situation has already been recorded in China for GM crops. Montsanto complained that in China its Bt cotton (Bollgard cotton) had undergone more severe biosafety tests and geographical limitations than the domestic Bt strains (Ho and Vermeer, 2004). At the Public Domain, the adoption of this application is strongly dependent on regional cultural and ethical values. For example, due to the strong aversion to GMOs and high animal welfare standards, this application is not expected to establish itself in the EU (Mora et al., 2011; Vàzquez-Salat et al., 2011). This is unlikely to cause any significant economic losses for either team as the EU is excluded -at least in the near futurefrom in their commercial strategy.

\section{CONCLUSION}

As seen in this article, the balance between benefits and risks can vary not only for each application but also at regional level. I have described the wide variety of factors that scientists need to take into account in order to ensure that GM animals reach the end user. The complex GMO regulatory set up provides a clear advantage for GM animals that enter vertically integrated sectors -dominated by MNCs- such as the pharmaceutical sector. However, in addition to the regulatory environment, there is a combination of cultural and socio-economic factors that will impact in each application. Some of these issues are general to the sector (i.e. dominance of MNCs in the healthcare sector) or to GM animals (i.e. long reproductive cycles increases the costs of developing funders) whereas others will be specific to each application (e.g. existing lactoferrin market in Asia). To illustrate how the different forces in Science, Market and Public Domains interact, I have provided an in-depth description of two GM applications: one that would enter the pharma sector (GM rabbits producing human polyclonal antibodies) and another for the food sector (GM cows producing human recombinant lactoferrin).

The pharmaceutical sector offers the most GM-friendly environment: benefits are clear, the market needs are tangible and, consequently, the public resistance is low. However, as seen with our case study, the positive environment does still not guarantee commercialisation. I have identified a series of obstacles that GM rabbits would encounter before reaching 
the patient community. Interestingly, the fact that some of them were technical (e.g. preference by rabbit B cells for the rabbit's heavy chain when producing antibodies) highlights the difficulties that scientists encounter when developing these applications. This case study proves the need to be cautious in projecting the promise of a technology before it is ready to reach the market.

On a broader context, I identified resistance from the pharmaceutical industry for these applications. The healthcare biotechnology sector is under strong pressure to produce cheaper and innovative drugs (Mestre-Ferrandiz et al., 2012). The GM rabbits are an excellent example of a novel application with promising therapeutic uses. Despite the clear needs from the patient community and the envisaged economic benefits linked to this type of technology, pharmaceutical companies were not inclined to leave their current market monopolies. With the existing governance of life sciences innovation, it might be left to the patient organisations to pressure governments for alternative pathways of commercialisation.

Conversely and despite entering the food sector, recombinant human lactoferrin from GM cows is likely to be approved by the regulatory authorities without attracting media attention. Their introduction to the market as tablets under the nutraceuticals umbrella will ensure that the product can be widely distributed without the additonal purification costs required by pharmaceutical standards. Nonetheless, once they reach the market this product will compete with an already established lactoferrin market. It is not clear whether the benefits of this application -cheaper upstream production and adaptability to the market demand- will be sufficient to ground itself in the market. In this case, the SKL might have a competitive advantage over Pharming by initially commercialising their product in the Chinese market. There is a clear need for the protective properties of lactoferrin and Chinese citizens are not as concerned about GMOs as other world regions (Zhang et al., 2010; Vàzquez-Salat and Salter, 2011). Public acceptance will vary regionally and both teams acknowledged that in regions such as the EU, this product is likely to find resistance from interest groups such as animal welfare organisations (Vàzquez-Salat and Salter, 2011). However, this was not identified as a major hurdle: neither team was planning to market their products in the EU. This is a particular shift from the GM plant scenario, where the EU (along with the USA) was a major global player.

Two main conclusions can be drawn from the results presented in this article. First, the differences seen in both case studies support the need to study GM animals on a case-by-case basis. Performing routinely this type of exercise at their early R\&D stage would allow researchers to tackle major limiting bottlenecks (e.g. consumer acceptance, market competition, regulatory set up, etc.) well in advance. This might prove particularly useful to facilitate the commercialisation of applications in controversial fields such as GM technology.

Second, one of the most significant changes seen in the GMO field is the array of states that have adopted GMOs. The GMO debate in the 90s was dominated by Western states, placing the US and EU in the opposite ends of the regulatory spectrum (Vàzquez-Salat et al., 2010). The increased investment in GMO research by some developing countries (e.g. Argentina, India, Philippines or China) has triggered a change in the international arena (Clive, 2012). This holds true not only for GM plants, but also for other related GMO applications such as GM animals. For example, whilst the USA placed itself as a global leader for GM plants, China and Argentina are believed to be the future global leaders in the GM animal field (Vàzquez-Salat and Houdebine, 2013).

At the same time, the stagnation of the EU's position on GM crops has isolated this region. This situation is only likely to worsen with the arrival of new GM applications such as GM animals. As E. Weise noted "Europe's opposition may be less significant in the future. China is now the biggest investor in public biotech crop research in the world" (www.usatoday.com/tech/ science/2010-03-17-Biotech17_cv_N.htm). It might be possible to consider a future where GM animals exist and the EU is at the driving seat of innovation. This scenario has already been acknowledged by the European Commission which is concerned for the loss of power of the EU in the global market. The EU's Strategy for Life Sciences and Biotechnology (2002) recommended developing forward-looking global policies in order to avoid being "confronted by policies shaped by others". Given the political history of GMOs in the EU, it is to be seen whether these recommendations can be translated into concrete actions.

Whilst it is difficult to imagine the end of the polarisation of the debate, the international GMO landscape is expected to change significantly in the near future. Hosting a higher number of players, the "newcomers" are likely to bring a different set of priorities to the discussion table. With the increasing importance in the global bioeconomy of pro-GM states (e.g. Argentina, China) and the lower profile by Western states on GM animals, there might be a global shift in GMO governance. It remains to be seen whether this change of actors will suffice to enhance the global adoption of both, GM animals and GM plants.

\section{ACKNOWLEDGEMENTS}

This article draws on research conducted by the author in the course of the PEGASUS programme. PEGASUS is an integrated project funded by the European Union in the Seventh Framework Programme (www.wageningenur.nl/en/ project/Pegasus.htm).

\section{REFERENCES}

BAGCHI-SEN S, SCULLY J (2007) Strategies and external relationships of small and medium-sized enterprises in the US agricultural biotechnology sector. Environment and Planning C-Government and Policy 25(6):844-860.

BUELOW R, VAN SCHOOTEN W (2007) The future of antibody therapy. Immunotherapy in 2020 4:83-106.

CANTLEY M (2007) An overview of regulatory tools and frameworks for modern biotechnology: A focus on agro-food. OECD International Futures Programme on "The Bioeconomy of 2030: Designing a Policy Agenda". Available at the World Wide Web: http://www.oecd.org/ futures / long-termtechnologicalsocietalchallenges / 40926623.pdf

CHAVATTE-PALMER P, CAMOUS S, JAMMES H LE CLEAC'H N, GUILLOMOT M, LEE RSF (2012) Review: Placental perturbations induce the developmental abnormalities often observed in bovine somatic cell nuclear transfer. Placenta, 33 Suppl, S99-S104.

CHEN KZ, ZHANG Y (2011) Regional case study: R2. Agricultural R\&D as an engine of productivity growth: China. Foresight Project on Global Food and Farming Futures.

CLIVE J (2012). Global Status of Commercialized Biotech/GM Crops: 2012. ISAAA Brief No. 44. ISAAA: Ithaca, NY.

COMMISSION COMMUNICATION "Life sciences and biotechnology A Strategy for Europe" $\operatorname{COM(2002)~} 27$ final. Available at the World 
Wide Web: http: / / eur-lex.europa.eu/LexUriServ/LexUriServ. do? uri=COM:2002:0027:FIN:EN:PDF

EUROBAROMETER (2005, June) Social values, science and technology. Special Eurobarometer 225/Wave 63.1. Brussels: TNS Opinion \& Social.

KLETER GA, VAN DER KRIEKEN WM, KOK EJ, BOSCH D, JORDI W, GILISSEN LJWJ (2001) Regulation and exploitation of genetically modified crops. Nature Biotechnology 19(12):1105-1110.

HO P, VERMEER EB (2004) Food safety concerns and biotechnology: Consumers' attitudes to genetically modified products in urban China. AgBioForum 7(4):158-175.

HOUDEBINE LM (2011) Production of human polyclonal antibodies by transgenic animals. Advances in Bioscience and Biotechnology 2: 138-141.

KOSTOV K, DJAMBAZOVA T, DIMOV G, VAN DIJK J, GROOT M, KLETER G, NOURDEM M, SMETS G, REDDY PG (2011, July) Life Sciences Dimension: Report on scenario development of GM animals and food (Work Package 4). Wageningen, Netherlands: PEGASUS (Public Perception of Genetically Modified Animals: Science, Utility and Society). Available on the World Wide Web: http://www.pegasus.wur. $\mathrm{nl} /$ UK/Documents/

LONNERDAL B, BRYANT A (2006) Absorption of iron from recombinant human lactoferrin in young US women. American Journal of Clinical Nutrition 83(2):305-309.

MESTRE-FERRANDIZ J, MORDOH A, SUSSEX J (2012) The many faces of Innovation. A report for the Association of the British Pharmaceutical Industry (ABPI) by the Office of Health Economics (OHE). Available on the World Wide Web: http://www.abpi.org.uk/our-work/library/ industry / Pages / many-faces-of-innovation.aspx

MORA C, MENOZZI D, ARAMYAN LH, VALEEVA NI, REDDY GP, ZIMMERMANN KL, MERIGO A, PANCINI A (2011, April) The Production chain context (Work Package 3, Deliverable 3.1). Wageningen, Netherlands: PEGASUS (Public Perception of Genetically Modified Animals: Science, Utility and Society). Available on the World Wide Web: http:/ / www.pegasus.wur.nl/UK/Documents/

NEETESON-VAN VIEWENHOVEN AM (1999) The future developments in farm animal breeding and reproduction and their ethical, legal and consumer implications. Report from the EC-ELSA project by Farm Animal Industrial Platform.

OECD (2009) The Bioeconomy to 2030: Designing a policy agenda. OECD International Futures Project. Available on the World Wide Web: http:// www.oecd.org/futures/bioeconomy/2030

ROYAL SOCIETY (2011) Knowledge, Networks and Nations: Global scientific collaborations in the $21^{\text {st }}$ century. Available on the World Wide Web: http:/ / royalsociety.org/policy/projects/knowledge-networksnations/report/
STEIN AJ, RODRÍGUEZ-CEREZO E (2008) Functional Food in the European Union. Report by the Joint Research Centre of the European Commission, EUR 23380 EN. Luxembourg: Office for Official Publications of the European Communities.

TAIT J (2007) Systemic interactions in life science innovation. Technology Analysis \& Strategic Management 19(3):257-277.

TANAKA K, IKEDA M, NOZAKI A, KATO N, TSUDA H, SAITO S, SEKIHARA H. (1999) Lactoferrin inhibits hepatitis C virus viremia in patients with chronic hepatitis C: A pilot study. Japanese Journal of Cancer Research 90(4):367-371.

TROOST FJ, SARIS WHM, BRUMMER RJM (2003) Recombinant human lactoferrin ingestion attenuates indomethacin-induced enteropathy in vivo in healthy volunteers. European Journal of Clinical Nutrition 57(12):1579-1585.

VÀZQUEZ-SALAT N, SALTER B and SMETS G. International multi-level governance of GMOs: The EU, USA and India cases (2010, September) (Work Package 6, Deliverable 6.1). Wageningen, Netherlands: PEGASUS (Public Perception of Genetically Modified Animals: Science, Utility and Society). Available on the World Wide Web: http://www.pegasus.wur. $\mathrm{nl} /$ UK/Documents/

VÀZQUEZ-SALAT N and SALTER B. Policy Implications of Introducing Genetically Modified (GM) Animals in the European Union (2011, October) (Work Package 6, Deliverable 6.2). Wageningen, Netherlands: PEGASUS (Public Perception of Genetically Modified Animals: Science, Utility and Society). Available on the World Wide Web: http://www. pegasus.wur.nl/UK/Documents/

VÀZQUEZ-SALAT N, SALTER B, SMETS G and HOUDEBINE LM (2012) The Current State of GMO Governance: Are We Ready for GM Animals? Biotechnology Advances.Nov;30(6):1336-43.

VÀZQUEZ-SALAT N and HOUDEBINE LM (2013) Will GM animals follow the GMO fate? Transgenic Research Feb;22(1):5-13.

VENKATESH MP, RONG L (2008) Human recombinant lactoferrin acts synergistically with antimicrobials commonly used in neonatal practice against coagulase-negative staphylococci and Candida albicans causing neonatal sepsis. Journal of Medical Microbiology 57(9): 1113-1121.

YANG P, WANG J, GONG G, SUN X, ZHANG R, DU Z, LIU Y, LI R, DING F, TANG B, DAI Y, LI N (2008) Cattle Mammary Bioreactor Generated by a Novel Procedure of Transgenic Cloning for Large-Scale Production of Functional Human Lactoferrin. Plos One 3(10).

ZHANG XY, HUAN JK, QIU HG, HUANG ZR (2010) A consumer segmentation study with regards to genetically modified food in urban China. Food Policy 35(5):456-462. 